\title{
Validation of a Novel Predictive Algorithm for Kidney Failure in Patients Suffering from Chronic Kidney Disease: The Prognostic Reasoning System for Chronic Kidney Disease (PROGRES-CKD)
}

\author{
Francesco Bellocchio ${ }^{1, *}$, Caterina Lonati ${ }^{2} \mathbb{D}$, Jasmine Ion Titapiccolo ${ }^{1}$, Jennifer Nadal ${ }^{3}$, Heike Meiselbach ${ }^{4}$, \\ Matthias Schmid ${ }^{3}$, Barbara Baerthlein ${ }^{5}$, Ulrich Tschulena ${ }^{6}$, Markus Schneider ${ }^{3}$, Ulla T. Schultheiss ${ }^{7,8}{ }^{10}$, \\ Carlo Barbieri ${ }^{6}$, Christoph Moore ${ }^{6}$, Sonja Steppan ${ }^{6}$, Kai-Uwe Eckardt ${ }^{4}, 9$, Stefano Stuard ${ }^{6}$ and Luca Neri ${ }^{1}$ (D)
}

1 Clinical \& Data Intelligence Systems-Advanced Analytics, Fresenius Medical Care Deutschland GmbH, 26020 Vaiano Cremasco, Italy; Jasmine.IonTitapiccolo@fmc-ag.com (J.I.T.); luca.neri@fmc-ag.com (L.N.)

2 Center for Preclinical Research, Fondazione IRCCS Ca' Granda Ospedale Maggiore Policlinico, 20122 Milan, Italy; caterina.lonati@gmail.com

3 Department of Medical Biometry, Informatics, and Epidemiology (IMBIE), Faculty of Medicine, University of Bonn, 53113 Bonn, Germany; Jennifer.Nadal@ukbonn.de (J.N.);

check for

updates

Citation: Bellocchio, F.; Lonati, C.; Ion Titapiccolo, J.; Nadal, J.;

Meiselbach, H.; Schmid, M.;

Baerthlein, B.; Tschulena, U.;

Schneider, M.; Schultheiss, U.T.; et al. Validation of a Novel Predictive Algorithm for Kidney Failure in Patients Suffering from Chronic Kidney Disease: The Prognostic Reasoning System for Chronic Kidney Disease (PROGRES-CKD). Int J. Environ. Res. Public Health 2021, 18 , 12649. https://doi.org/10.3390/ ijerph182312649

Academic Editor: Paul B. Tchounwou

Received: 28 October 2021

Accepted: 25 November 2021

Published: 30 November 2021

Publisher's Note: MDPI stays neutral with regard to jurisdictional claims in published maps and institutional affiliations.

Copyright: (c) 2021 by the authors. Licensee MDPI, Basel, Switzerland. This article is an open access article distributed under the terms and conditions of the Creative Commons Attribution (CC BY) license (https:/ / creativecommons.org/licenses/by/ $4.0 /)$. matthias.schmid@imbie.uni-bonn.de (M.S.); markus.schneider@extern.uk-erlangen.de (M.S.)

4 Department of Nephrology and Hypertension, Friedrich-Alexander University of Erlangen-Nürnberg, 91054 Erlangen, Germany; Heike.Meiselbach@uk-erlangen.de (H.M.); kai-uwe.eckardt@charite.de (K.-U.E.)

5 Medical Centre for Information and Communication Technology (MIK), University Hospital Erlangen, 91054 Erlangen, Germany; Barbara.Baerthlein@uk-erlangen.de

6 Fresenius Medical Care, Deutschland GmbH, 61352 Bad Homburg, Germany; Ulrich.Tschulena@fmc-ag.com (U.T.); carlo.barbieri@fmc-ag.com (C.B.); Christoph.Moore@fmc-ag.com (C.M.); Sonja.Steppan@fmc-ag.com (S.S.); Stefano.stuard@fmc-ag.com (S.S.)

7 Institute of Genetic Epidemiology, Faculty of Medicine and Medical Center, University of Freiburg, 79085 Freiburg, Germany; ulla.schultheiss@uniklinik-freiburg.de

8 Department of Medicine IV-Nephrology and Primary Care, Faculty of Medicine and Medical Center, University of Freiburg, 79085 Freiburg, Germany

9 Department of Nephrology and Medical Intensive Care, Charité Universitätsmedizin Berlin, 10117 Berlin, Germany

* Correspondence: francesco.bellocchio@fmc-ag.com

Abstract: Current equation-based risk stratification algorithms for kidney failure (KF) may have limited applicability in real world settings, where missing information may impede their computation for a large share of patients, hampering one from taking full advantage of the wealth of information collected in electronic health records. To overcome such limitations, we trained and validated the Prognostic Reasoning System for Chronic Kidney Disease (PROGRES-CKD), a novel algorithm predicting end-stage kidney disease (ESKD). PROGRES-CKD is a naïve Bayes classifier predicting ESKD onset within 6 and 24 months in adult, stage 3-to-5 CKD patients. PROGRES-CKD trained on 17,775 CKD patients treated in the Fresenius Medical Care (FMC) NephroCare network. The algorithm was validated in a second independent FMC cohort $(n=6760)$ and in the German Chronic Kidney Disease (GCKD) study cohort $(n=4058)$. We contrasted PROGRES-CKD accuracy against the performance of the Kidney Failure Risk Equation (KFRE). Discrimination accuracy in the validation cohorts was excellent for both short-term (stage 4-5 CKD, FMC: AUC = 0.90, 95\%CI 0.88-0.91; GCKD: AUC $=0.91,95 \%$ CI 0.86-0.97) and long-term (stage 3-5 CKD, FMC: AUC = 0.85, 95\%CI 0.83-0.88; GCKD: AUC $=0.85,95 \% C I$ 0.83-0.88) forecasting horizons. The performance of PROGRES-CKD was non-inferior to KFRE for the 24-month horizon and proved more accurate for the 6-month horizon forecast in both validation cohorts. In the real world setting captured in the FMC validation cohort, PROGRES-CKD was computable for all patients, whereas KFRE could be computed for complete cases only (i.e., $30 \%$ and $16 \%$ of the cohort in 6- and 24-month horizons). PROGRES-CKD accurately predicts KF onset among CKD patients. Contrary to equation-based scores, PROGRESCKD extends to patients with incomplete data and allows explicit assessment of prediction robustness in case of missing values. PROGRES-CKD may efficiently assist physicians' prognostic reasoning in real-life applications. 
Keywords: chronic kidney disease (CKD); end-stage kidney disease (ESKD); kidney replacement therapy (KRT); risk prediction; artificial intelligence; machine learning; naïve Bayes classifiers; precision medicine

\section{Introduction}

Multiple behavioral and pharmacological interventions have proven effective in reducing the burden of risk factors for chronic kidney disease (CKD) progression [1-4]. Furthermore, timely transition management (i.e., vascular access creation and training) for patients needing Kidney Replacement Therapy (KRT) is associated with prolonged survival and reduced complication rates once on dialysis, while delayed referrals are associated with increased morbidity, mortality, and healthcare costs [5], as well as worse patient quality of life [6]. Therefore, early identification of high risk patients is an essential prerequisite of personalized clinical decision making [7-9].

Several prediction models were developed to assist physicians in forecasting CKD progression [10]. However, most of them have not been consistently implemented in clinical practice $[9,11,12]$. Indeed, the majority of published risk scores lack external validation $[11,13,14]$, leading to suboptimal discrimination in external populations [12] and limited generalizability to clinical settings [11]. One prominent exception is represented by the Kidney Failure Risk Equations (KFREs) developed by Tangri and colleagues [15], which showed stable discrimination in different validation studies [16-18]. However, KFREs do not provide short-term forecasts, are not calculable for patients with incomplete data, and need re-calibration when applied to CKD populations with risk factor distributions departing from those of the original derivation dataset.

To overcome such limitations, we developed the Prognostic Reasoning System for Chronic Kidney Disease (PROGRES-CKD), a risk score application for adult patients suffering from CKD stages 3-5. PROGRES-CKD is based on a naïve Bayes Classifier (NBC) algorithm and it was trained on a large-multinational clinical dataset, reflecting real-world clinical practice. The application includes PROGRES-CKD-6 for 6-month forecasting and PROGRES-CKD-24 for 24-month forecasting.

In the present study, we reported the training and validation of both PROGRES-CKD-6 and PROGRES-CKD-24 in two independent samples of CKD patients: the FMC NephroCare cohort (European Clinical Database, EuCliD ${ }^{\circledR},[19,20]$ ) and the German Chronic Kidney Disease (GCKD) study cohort [21]. Moreover, we compared the PROGRES-CKD discrimination accuracy and suitability for clinical practice against the KFREs equations.

\section{Materials and Methods}

In reporting PROGRES-CKD training and validation studies we adhered to the Transparent reporting of a multivariable prediction model for individual prognosis or diagnosis (TRIPOD) statement [22] and to the Guidelines for Developing and Reporting Machine Learning Predictive Models in Biomedical Research [23].

\subsection{Description of Naïve Bayes Classifiers}

All PROGRES-CKD models are NBCs. NBCs are probabilistic models based on application of the Bayes' theorem. The basic assumption of NBCs is conditional independence of predictors given the outcome. NBCs are represented through directed acyclic graphs (Figure 1). NBCs have been previously used in medical applications for diagnostic and prognostic reasoning in several therapeutic areas [24,25]. In fact, once derived and validated, NBCs generate metrics informing medical prognostic reasoning. First, they generate a risk score representing the expected incidence of a disease/event given a vector of known patient characteristics. Furthermore, NBCs can be used to generate value of information (VOI) statistics and impact metrics. VOI statistics represent the reduction in uncertainty (i.e., entropy) in the outcome variable that would be obtained had the value of missing 
variables been observed instead [26]. Therefore, it can be used to prioritize additional diagnostic testing or biomarker assays for patients with incomplete medical records. Third, NBCs can provide impact metrics (i.e., Normalized Likelihood (NL) [27]) for each observed variable. Impact metrics can be interpreted as the magnitude of association of different subsets of evidence on the outcome variable.

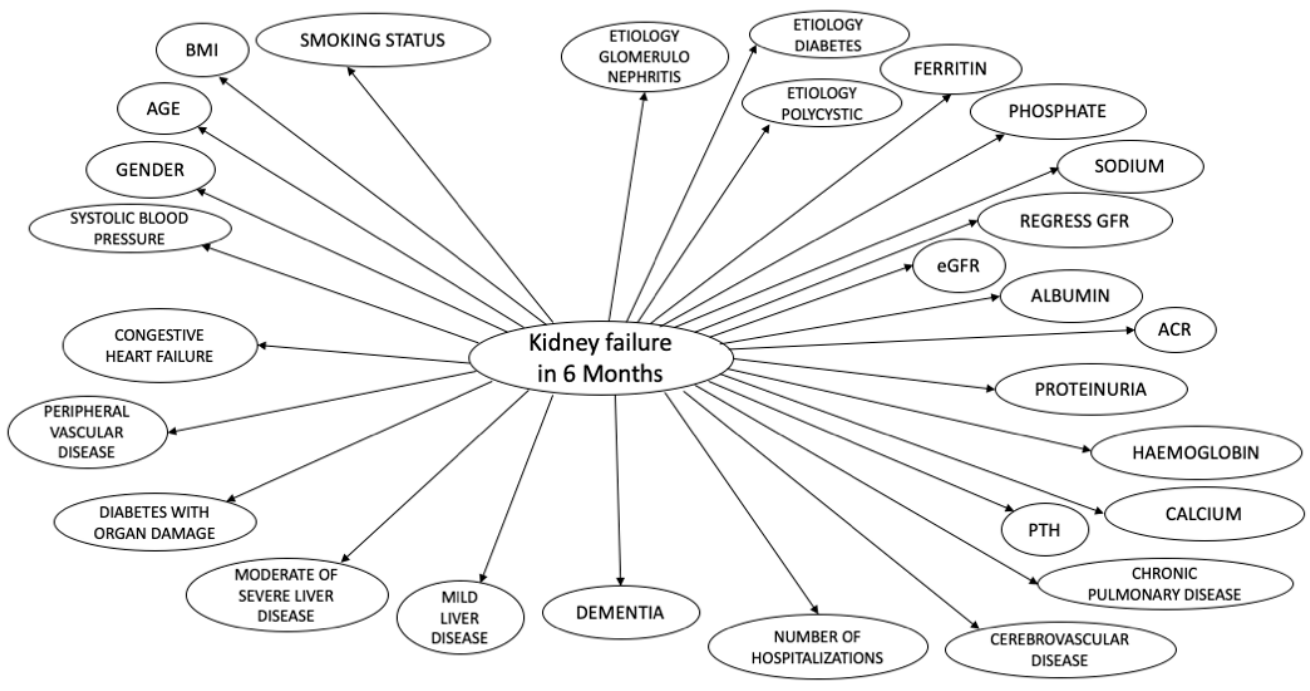

(a)

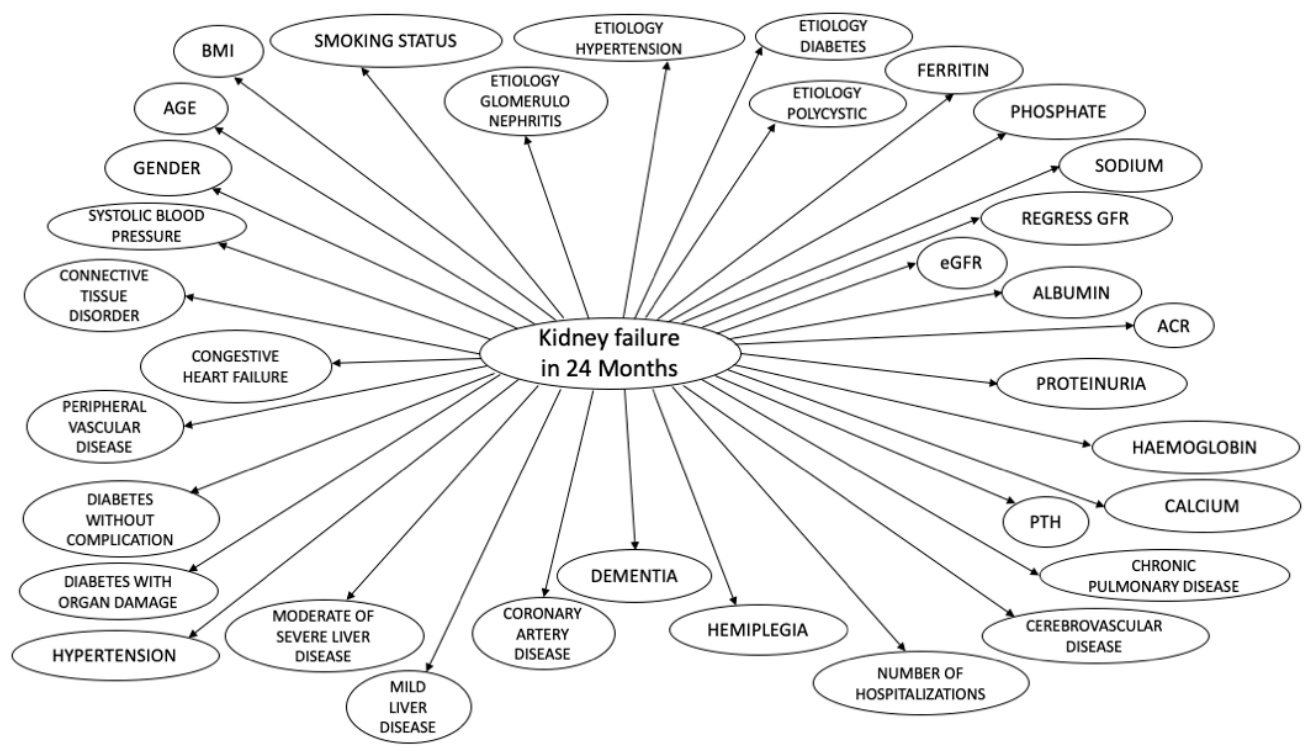

(b)

Figure 1. The Bayesian Network structure of PROGRES-CKD. (a) PROGRESS-CKD-6; (b) PROGRESSCKD-24.

\subsection{PROGRES-CKD Training}

In this application of NBCs, we aimed at developing a model to predict the risk of KRT initiation within 6 and 24 months. The risk score is anchored at $0.00=$ no risk at all to $1.00=$ certainty of failure within the prediction horizons .

We derived model weights for the PROGRES-CKD by a data-driven algorithm, exploiting the wealth of information collected in the European Clinical Database $\left(\mathrm{EuCliD}^{\circledR}\right.$, Fresenius Medical Care Deutschland $\mathrm{GmbH}$, Bad Homburg, Germany), a large, multinational, database of CKD patients. All nephrology clinics belonging to the Fresenius Medical Care (FMC) NephroCare network confer data collected for healthcare practice into this cen- 
tralized data-repository. EuCliD ${ }^{\circledR}$ is a fully codified database recording clinical, laboratory, socio-demographic, treatment and prescription data for each medical encounter $[19,20]$. Information is collected by healthcare professionals either manually or by means of interfaces to existing local data managing systems.

All non-dialysis dependent, stage 3-5 CKD patients receiving care in outpatient renal clinics belonging to the NephroCare network from 2017 to 2018 were screened for eligibility. We enrolled only patients who received at least one outpatient visit and one serum creatinine (s-cr) assessment. The endpoints of interest were KRT initiation within 6 and 24 months. We excluded patients dying before reaching the endpoint or before the end-of-follow-up (i.e., 6 or 24 months, depending on endpoint of interest). Overall, 22,535 subjects met the inclusion criteria. This initial dataset was randomly partitioned into 2 analytical samples: development $(70 \%, n=17,775)$, and validation $(30 \%, n=6760)$. The derivation of NBC weights was obtained with Hugin 8.5.

\subsection{Measures}

\subsubsection{Endpoint Definition}

The primary endpoint was KRT initiation within 6 and 24 months. Outcome definition does not include episodes of dialysis treatment for acute and transient kidney derangement.

We defined patients as "lost to follow" when no additional s-cr assessments after end of follow-up date and no dialysis-dependence onset notes were present in the clinical records.

\subsubsection{Input Variables}

A list of all the variables included in the final model is provided in Table 1. The final model for the 6-month forecast incorporates 28 independent variables, while the model for the 24-month forecast includes 34 variables.

Table 1. Variables included in PROGRES-CKD models.

\begin{tabular}{|c|c|c|c|}
\hline & & PROGRES-CKD-6 & PROGRES-CKD-24 \\
\hline Group & Variable & $n=28$ & $n=34$ \\
\hline \multicolumn{4}{|c|}{ Demographics and anthropometrics } \\
\hline & Age & $X$ & $X$ \\
\hline & Gender & $X$ & $X$ \\
\hline & BMI, $\mathrm{Kg} / \mathrm{m}^{2}$ & $x$ & $X$ \\
\hline & Smoking status & $X$ & $x$ \\
\hline \multicolumn{4}{|c|}{ Kidney function } \\
\hline & Albumin, g/dL & $x$ & $x$ \\
\hline & $\begin{array}{l}\text { Albumin Creatinine Ratio (ACR), } \\
\mathrm{mg} / \mathrm{mmol}^{* *}\end{array}$ & $x$ & $x$ \\
\hline & Calcium, mg/dL & $x$ & $x$ \\
\hline & $\mathrm{eGFR},\left(\mathrm{ml} / \mathrm{min} / 173 \mathrm{~m}^{2}\right)$ & $X$ & $X$ \\
\hline & regressGFR * & $x$ & $x$ \\
\hline & Hemoglobin, g/dL & $x$ & $X$ \\
\hline & Phosphate, $\mathrm{mg} / \mathrm{dL}$ & $x$ & $x$ \\
\hline & Urine protein, $\mathrm{g} / 24 \mathrm{~h}$ & $X$ & $X$ \\
\hline & Parathyroid hormone, ng/L & $x$ & $x$ \\
\hline & Sodium, $\mathrm{mmol} / \mathrm{L}$ & $x$ & $x$ \\
\hline & Ferritin, microg/L & $X$ & $X$ \\
\hline \multicolumn{4}{|c|}{ Etiology of kidney disease } \\
\hline & Diabetes & $x$ & $x$ \\
\hline & Hypertension & & $x$ \\
\hline & Glomerulonephritis & $x$ & $x$ \\
\hline & Polycystic & $x$ & $x$ \\
\hline
\end{tabular}


Table 1. Cont.

\begin{tabular}{|c|c|c|c|}
\hline & & PROGRES-CKD-6 & PROGRES-CKD-24 \\
\hline Group & Variable & $n=28$ & $n=34$ \\
\hline \multicolumn{4}{|c|}{ Comorbidities } \\
\hline & Cerebrovascular disease & $x$ & $x$ \\
\hline & Chronic Pulmonary Disease & $x$ & $x$ \\
\hline & Congestive heart failure & $x$ & $x$ \\
\hline & Connective tissue disorder & & $X$ \\
\hline & Coronary artery disease & & $x$ \\
\hline & Dementia & $X$ & $x$ \\
\hline & Diabetes with organ damage & $X$ & $X$ \\
\hline & Diabetes without complications & & $x$ \\
\hline & Hemiplegia & & $X$ \\
\hline & Hypertension & & $x$ \\
\hline & Mild liver disease & $x$ & $x$ \\
\hline & Moderate or severe liver disease & $x$ & $x$ \\
\hline & Peripheral vascular disease & $x$ & $x$ \\
\hline \multicolumn{4}{|l|}{ Other } \\
\hline & Number of hospitalizations & $x$ & $x$ \\
\hline & Systolic blood pressure & $X$ & $x$ \\
\hline
\end{tabular}

* Slope of linear regression of eGFR values over the last 12 months. ${ }^{* *}$ Urine Protein-Creatinine Ratio was converted to ACR by ACR = Urin protein*PCR (Urine protein $=0.6)$ (please, see the Supplementary Material for the conversion table).

We assessed demographic, anthropometric, and lifestyle variables at index visit; blood biomarkers were collected and averaged over 12 months before index date (i.e., during the ascertainment period); their slope (i.e., change rate) was likewise calculated. Lifetime occurrence of comorbidities was evaluated by abstracting ICD10 codes [28] from outpatient medical records (Supplementary Material). Finally, etiologies of kidney disease were also noted.

\subsubsection{Definition of CKD Stages}

GFR was estimated in adults using the 2009 CKD-EPI creatinine equation [29]. Patients are classified into one of the following GFR categories: (1) G1 normal or high, GFR: $\geq 90 \mathrm{~mL} / \mathrm{min} / 1.73 \mathrm{~m}^{2}$; (2) G2 mildly decreased, GFR: $60-89 \mathrm{~mL} / \mathrm{min} / 1.73 \mathrm{~m}^{2}$; (3) G3a mildly to moderately decreased, GFR: 45-59; (4) G3b moderately to severely decreased, GFR: 30-44; (5) G4 severely decreased, GFR: 15-29; (6) G5 kidney failure, GFR: <15.3.

\subsection{Design and Setting of PROGRES-CKD Validation Studies}

For the validation study we randomly selected one visit from patients' histories (index date) before occurrence of study endpoint. All information collected before the index data was used as an input variable for the model. Patients dying before reaching the endpoint or before the end-of-follow-up (i.e., 6 or 24 months, depending on endpoint of interest) were excluded.

Based on this general design setting, we validated PROGRES-CKD models in two independent cohorts.

\subsubsection{Study A}

The first validation study was performed in the testing cohort derived from $30 \%$ partitioning of the clinical data abstracted from the FMC NephroCare cohort.

\subsubsection{Study B}

A second analysis evaluated PROGRES-CKD performance using data from the German CKD study [21]. Briefly, the GCKD study is an ongoing prospective observational national study that recruited 5217 patients with CKD of various etiologies. The enrolment period started in July 2011 and ended in 2012. Patient recruitment and follow-up is orga- 
nized through a network of academic nephrology centers collaborating with practicing nephrologists throughout Germany. The main study endpoints were mortality, decline in kidney function, and cardiovascular events. At the time of recruitment, patients were under nephrological care and showed either eGFR of $30-60 \mathrm{~mL} / \mathrm{min} / 1.73 \mathrm{~m}^{2}$ or overt urin protein in the presence of an eGFR $>60 \mathrm{~mL} / \mathrm{min} / 1.73 \mathrm{~m}^{2}$. In our validation analysis, only patients subjected to serum creatinine evaluation at baseline and followed for at least 2 years were considered.

\subsubsection{Study C}

We conducted an impact study assessing concordance of nephrologists' and PROGRESCKD-24 ratings of risk. Four experts were asked to forecast KRT initiation risk for 78 CKD patients based on their demographic, anthropometric, and clinical data. These patients were randomly selected from the FMC NephroCare cohort and had complete clinical history up to 24 months after the index date. Information related to all input variables used by the model were extracted from existing clinical records. Information extracts for each patient were collected in real-world clinical practice by physicians during outpatient visits. Doctors were asked to rate KRT risk on a 10-point rating scale anchored at 1 (risk is negligible, almost no patient with these characteristics would require RRT within 2 years), 5 (about $50 \%$ of patients with these characteristics would require RRT within 2 years) and 10 (almost $100 \%$ patients with these characteristics would require RRT within 2 years). Risk ratings provided by the physicians were then compared to scores obtained from PROGRES-CKD-24 for the same patients. Comparative analysis included accuracy, sensitivity, and specificity based on score cut-off that maximized Youden's Index. Thereafter, we investigated the potential impact of using risk scores provided by either experts or PROGRES-CKD-24 in referring patterns to intensified healthcare prevention programs aimed at delaying CKD progression. We simulated the use of risk estimates on a large, hypothetical CKD population of stage 3-5 CKD patients $(n=10,000)$, assuming an ESRD incidence within 24 months of $4.6 \%$ (i.e., $n=460$ expected ESKD cases) and an intervention effect size of 1.5 (i.e., patients in the standard of care arm would face 50\% higher risk of ESKD compared to those allocated in the intensified healthcare program). The intervention effect size was estimated based on expert opinion and several intensified intervention programs reported in diabetic and non-diabetic CKD [30-32].

\subsection{Statistical Analysis}

We computed the cumulative incidence and the incidence density of KRT initiation events in the study population and their 95\% confidence intervals based on the Poisson distribution.

Since PROGRES-CKD models are NBCs, no data manipulation was required to explicitly handle missing variables.

Model performance was evaluated by concordance statistic and calibration charts in the FMC NephroCare and the GCKD cohorts. Discrimination was quantified by calculating the area under the receiver operating characteristic curve (ROC AUC) [33]. An AUC $>0.70$ was considered acceptable. Calibration was visually inspected by plotting observed outcome incidence by quintiles of the risk score [34].

A further analysis investigated non-inferiority (defined as $\triangle \mathrm{AUC}<0.05$ ) of both PROGRES-CKD-6 and PROGRES-CKD-24 relative to the KFREs [15] calibrated for the European population [16]. Briefly, Tangri's models were developed using Cox proportional hazards regression methods in stage 3-5 CKD patients. In the present study, the following Tangri's equations were used: (1) 4 Variables (4VAR), includes Age, Gender, eGFR, and Albumin-Creatinine Ratio (ACR); (2) 6 Variables (6VAR), includes Age, Gender, eGFR, ACR, Diabetes, and Hypertension. We could not apply the 8 Variables (8VAR) equation given the lack of serum bicarbonate assessments in both study cohorts. Non-inferiority was assessed by checking whether a one-sided confidence interval of the AUC remained entirely above the non-inferiority threshold (0.05). In case non-inferiority was achieved, we evaluated superiority of PROGRES-CKD compared to benchmark models; superiority 
was set at $\triangle \mathrm{AUC} \geq 0.05$. Given the sequential nature of testing in a fixed order method approach, type I error is not inflated by multiple testing. Superiority was tested with the DeLong non-parametric approach [35]. Statistical significance was claimed at $\alpha<0.05$.

For study C, the following accuracy parameters were considered: Sensitivity, Specificity, Positive Predictive Value (PPV), and False Omission Rate (FOR). We also calculated the number needed to treat (NNT) in order to avoid $1 \mathrm{KRT}$ event as the reciprocal of the absolute risk difference between the hypothetical prevention program and standard of care for all patients:

$\mathrm{NNT}=(\#$ patients int tr $/[(\#$ patients int tr $* \mathrm{PPV})-((\#$ patients int $\operatorname{tr} * \mathrm{PPV}) /($ effect - size $))]$

Model training was performed using Hugin Explorer. All analyses for the validation study were performed with SAS $9.4^{\circledR}$.

\section{Results}

\subsection{Cohort Characteristics}

Table 2 reports baseline demographic and clinical data of the whole FMC NephroCare cohort. Among 22,535 non-dialysis-dependent stage 3-5 CKD patients, 18,504 and 9407 patients had 6 and 24 months of follow-up, respectively. KRT events were 801 within 6 months (8.66 events /100 person-year) and 1817 within 24 months (9.66 events/100 person-year). On the other hand, KRT events in the validation sample (derived from $30 \%$ partitioning of the whole FMC cohort) were 248 (2.24 events / 100 person-year) and 537 (9.36 events/ 100 person-year) within 6 and 24 months, respectively.

Table 2. Baseline characteristics of patients from the FMC NephroCare and GCKD cohorts.

\begin{tabular}{|c|c|c|c|c|}
\hline \multirow[b]{2}{*}{ Variable } & \multicolumn{2}{|c|}{ FMC Cohort } & \multicolumn{2}{|c|}{ GCKD Cohort } \\
\hline & $n$ & $\begin{array}{l}\text { Mean } \pm \text { SD or Median } \\
\text { (IQR) or } n(\%)\end{array}$ & $n$ & $\begin{array}{c}\text { Mean } \pm \text { SD or Median } \\
(I Q R) \text { or } n(\%)\end{array}$ \\
\hline Stage 3 & 11,965 & $11,965(53.1 \%)$ & 3593 & $3593(88.54 \%)$ \\
\hline Stage 4 & 8026 & $8026(35.62 \%)$ & 460 & $460(11.34 \%)$ \\
\hline Stage 5 & 2544 & $2544(11.29 \%)$ & 5 & $5(0.12 \%)$ \\
\hline Age (year) & 22,535 & $72.15 \pm 11.7$ & 4058 & $62.12 \pm 10.50$ \\
\hline BMI $\left(\mathrm{kg} / \mathrm{cm}^{2}\right)$ & 21,655 & $30.63 \pm 10.92$ & 4015 & $30.03 \pm 5.91$ \\
\hline $\operatorname{eGFR}\left(\left(\mathrm{mL} / \mathrm{min} / 1.73 \mathrm{~m}^{2}\right)\right.$ & 22,535 & $31.93 \pm 13.4$ & 4058 & $41.92 \pm 9.76$ \\
\hline Albumin (g/dL) & 19,004 & $4.19 \pm 0.4$ & 4055 & $3.85 \pm 0.42$ \\
\hline Ferritin $(\mu \mathrm{g} / \mathrm{L})$ & 7303 & $222.18 \pm 260.98$ & 1044 & $200.48 \pm 196.11$ \\
\hline Hemoglobin (g/dL) & 21,916 & $12.65 \pm 1.83$ & 3978 & $13.49 \pm 1.69$ \\
\hline Phosphate (mg/dL) & 20,362 & $3.65 \pm 0.74$ & 4058 & $3.45 \pm 0.64$ \\
\hline Calcium (mg/dL) & 20,686 & $9.36 \pm 0.73$ & 4058 & $9.07 \pm 0.63$ \\
\hline Sodium (mmol/L) & 20,612 & $140.17 \pm 3.16$ & 4057 & $139.70 \pm 3.14$ \\
\hline PTH (ng/L) & 9466 & $131.84 \pm 150.12$ & 0 & - \\
\hline $\mathrm{ACR}(\mathrm{mg} / \mathrm{mmol})$ & 90 & $138.67 \pm 568.28$ & 3999 & $393.63 \pm 888.48$ \\
\hline Proteinuria $(\mathrm{g} / 24 \mathrm{~h})$ & 8780 & $3.58 \pm 150.29$ & 0 & - \\
\hline Systolic (mmHg) & 17,963 & $137.33 \pm 18.41$ & 4030 & $140.27 \pm 20.53$ \\
\hline $\mathrm{CRP}(\mathrm{mg} / \mathrm{L})$ & 13,468 & $4.23(7.63)$ & 4056 & $2.41(4.27)$ \\
\hline Glucose (mg/dL) & 19,499 & $126.45 \pm 48.59$ & 0 & - \\
\hline HDL Cholesterol (mg/dL) & 7074 & $48.3 \pm 16.74$ & 4051 & $50.72 \pm 17.35$ \\
\hline LDL Cholesterol (mg/dL) & 7084 & $107.59 \pm 219.29$ & 4051 & $116.33 \pm 42.93$ \\
\hline Triglyceride (mg/dL) & 15,191 & $142.77(95.72)$ & 4050 & $173.38(126.45)$ \\
\hline hsTNT (ng/L) & 0 & - & 3976 & $13(11)$ \\
\hline Uric Acid (mg/dL) & 20,273 & $6.68 \pm 1.61$ & 4058 & $7.40 \pm 1.92$ \\
\hline
\end{tabular}


Table 2. Cont

\begin{tabular}{|c|c|c|c|c|}
\hline \multirow[b]{2}{*}{ Variable } & \multicolumn{2}{|c|}{ FMC Cohort } & \multicolumn{2}{|c|}{ GCKD Cohort } \\
\hline & $n$ & $\begin{array}{c}\text { Mean } \pm \text { SD or Median } \\
(\text { IQR) or } n(\%)\end{array}$ & $n$ & $\begin{array}{c}\text { Mean } \pm \text { SD or Median } \\
(\text { IQR) or } n(\%)\end{array}$ \\
\hline Gender (M) & 22,535 & $11,349(50.36 \%)$ & 4058 & $2510(61.85 \%)$ \\
\hline Etiology Diabetes & 22,535 & $3614(16.04 \%)$ & 4058 & $666(16.41 \%)$ \\
\hline Etiology Polycystic & 22,535 & $477(2.12 \%)$ & 4058 & $157(3.87 \%)$ \\
\hline Etiology Hypertension & 22,535 & $5281(23.43 \%)$ & 4058 & $1011(24.91 \%)$ \\
\hline Etiology Glomerulonephrite & 22,535 & $987(4.38 \%)$ & 4058 & $623(15.35 \%)$ \\
\hline Smoking status: ex-smoker & 3502 & $3502(15.54 \%)$ & 1819 & $1819(44.96 \%)$ \\
\hline Smoking status: no smoker & 10,066 & $10,066(44.67 \%)$ & 1649 & $1649(40.76 \%)$ \\
\hline Smoking status: smoker & 2274 & $2274(10.09 \%)$ & 578 & $578(14.29 \%)$ \\
\hline Alcohol: abuse & 8636 & $8636(38.32 \%)$ & 771 & $771(19.10 \%)$ \\
\hline Alcohol: moderate & 0 & $0(0 \%)$ & 3265 & $3265(80.90 \%)$ \\
\hline Alcohol: abstinence & 6984 & $6984(30.99 \%)$ & 0 & $0(\%)$ \\
\hline Peripheral Vascular Disease & 22,535 & $1875(8.32 \%)$ & 4058 & $424(10.45 \%)$ \\
\hline Coronary Artery Disease & 22,535 & $4336(19.24 \%)$ & 4058 & $908(22.38 \%)$ \\
\hline Congestive Heart Failure & 22,535 & $1887(8.37 \%)$ & 4058 & $776(19.12 \%)$ \\
\hline Cerebrovascular Disease & 22,535 & $1876(8.32 \%)$ & 4058 & $472(10.52 \%)$ \\
\hline Connective Tissue Disorder & 22,535 & $399(1.77 \%)$ & 0 & - \\
\hline Cancer & 22,535 & $2469(10.96 \%)$ & 4058 & $532(13.11 \%)$ \\
\hline Diabetes & 22,535 & $9021(40.03 \%)$ & 4058 & $1545(38.07 \%)$ \\
\hline Anemia & 22,535 & $9800(43.49 \%)$ & 4058 & $1057(26.05 \%)$ \\
\hline Hypertension & 22,535 & $17,871(79.3 \%)$ & 4058 & $3951(97.36 \%)$ \\
\hline Atrial Fibrillation & 22,535 & $2337(10.37 \%)$ & 4058 & $876(21.59 \%)$ \\
\hline Diabetes Without Complications (CCI) & 22,535 & $3013(13.37 \%)$ & 4058 & $1545(38.07 \%)$ \\
\hline Chronic Pulmonary Disease (CCI) & 22,535 & $1618(7.18 \%)$ & 4058 & $285(7.02 \%)$ \\
\hline Psychiatric Disease & 22,535 & $177(0.79 \%)$ & 0 & - \\
\hline Liver Disease & 22,535 & $987(4.38 \%)$ & 0 & - \\
\hline RRT in 24 months & 9407 & $1817(19.32 \%)$ & 3684 & $80(2.17 \%)$ \\
\hline RRT in 6 months & 18,504 & $801(4.33 \%)$ & 3888 & $11(0.28 \%)$ \\
\hline
\end{tabular}

A second validation study was performed using data from the GCKD study. As shown in Table 2, a total of 4058 stage 3-5 CKD patients were included, of whom 3888 and 3687 subjects had 6 and 24 months of follow-up, respectively. RRT events were 11 within 6 months ( 0.5 events $/ 100$ person-year) and 80 (1.1 events / 100 person-year) within 24 months.

Early CKD stages were predominantly represented in the GCKD study, whereas patients in stage 5 CKD were mostly enrolled in the FMC NephroCare cohort. Loss to follow-up within 6 months was $4031(17.9 \%)$ and $170(4.2 \%)$ participants, while loss to follow-up in 24 months was 13,128 (58.3\%) and 371 (9.1\%) participants in the FMC NephroCare and GCKD cohorts, respectively.

\subsection{Model Discrimination in the Training and Validation Dataset from the FMC NephroCare Cohort}

In the development dataset, AUC of PROGRES-CKD-6 was 0.88 (95\%CI 0.86-0.89) in stage $4-5$ patients, while AUC of PROGRES-CKD-24 was $0.86(95 \%$ CI $0.85-0.87)$ in stage $3-5$ patients.

External validation was performed in an independent sample of patients treated in the FMC NephroCare cohort. Analysis indicated a good discriminative ability for both PROGRES-CKD-6 and PROGRES-CKD-24 models, with a concordance statistic of 0.90 (95\%CI 0.88-0.91, stage 4-5) and 0.85 (95\%CI 0.83-0.88, stage 3-5), respectively.

Calibration of predicted versus observed risk is represented in Figure 2. 
(A)

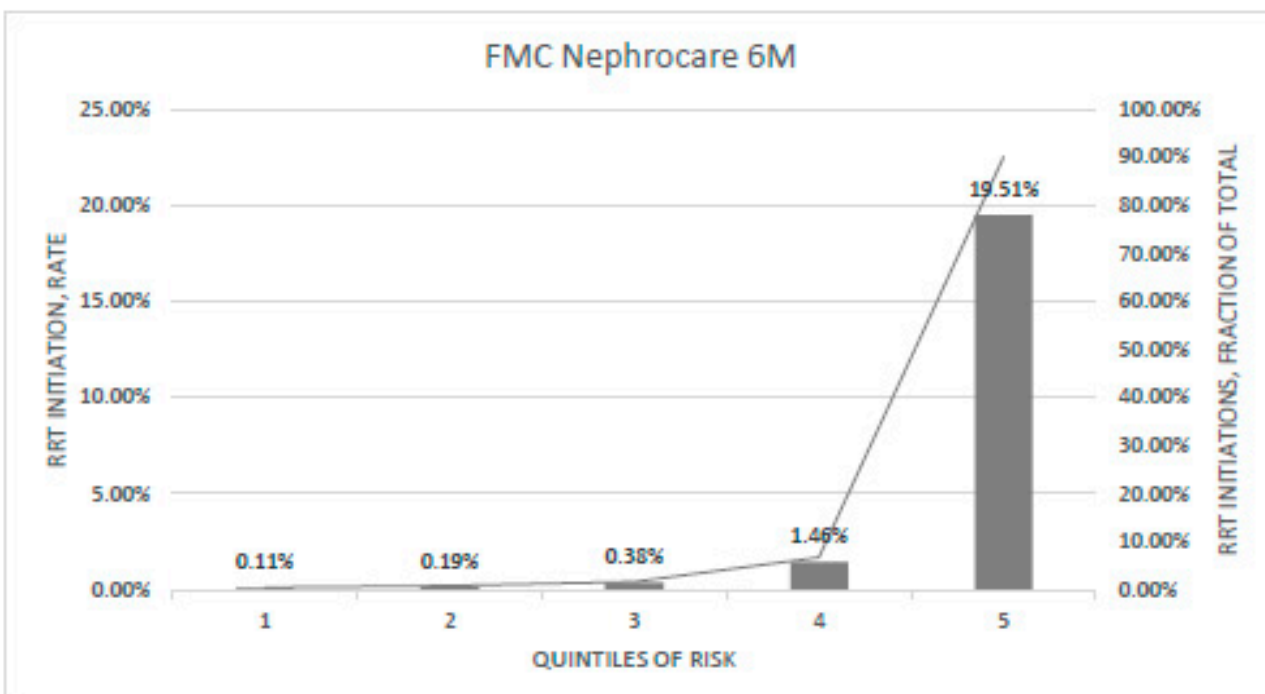

(B)

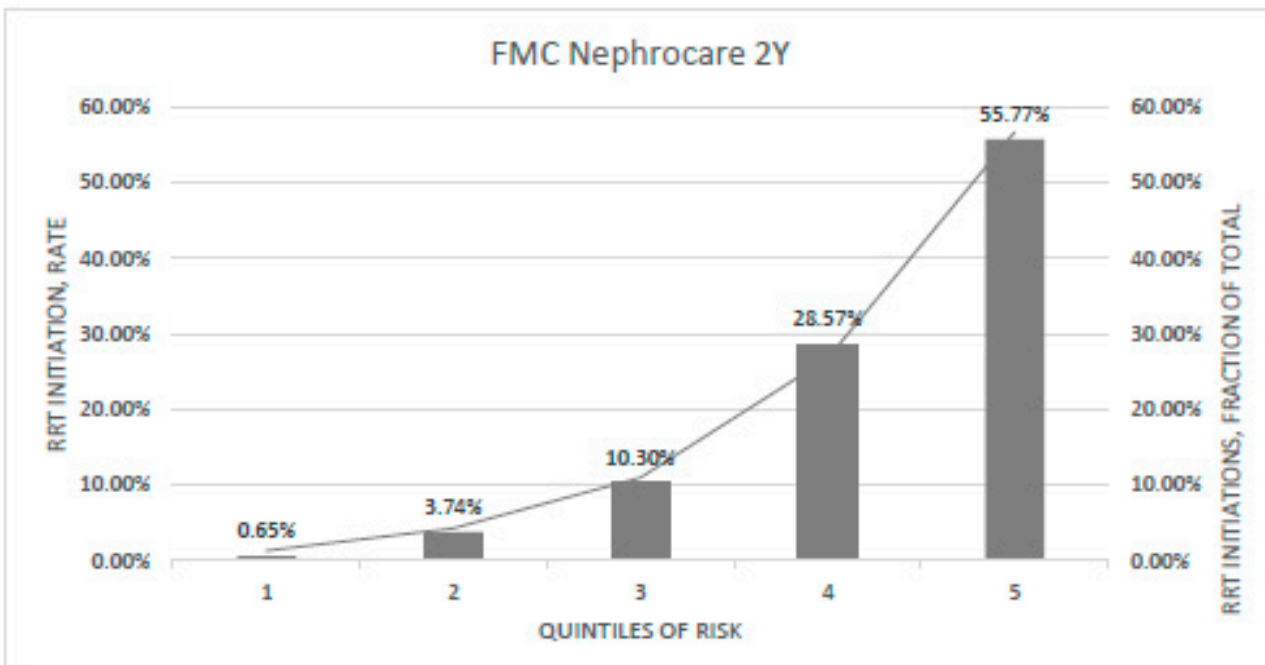

Figure 2. Calibration of (A) PROGRES-CKD-6, and (B) PROGRES-CKD-24 in the FMC cohort. Bar graph denotes the incidence of RRT initiation events observed in each quintile of risk (left axis); line graph denotes the fraction of RRT initiation events in each quintile with respect to the total number of RRT initiation events (right axis). Endpoint horizons: 6 months for PROGRES-CKD-6; 24 months for PROGRES-CKD-24.

\subsection{Model Discrimination in the GCKD Cohort}

PROGRES-CKD models showed a good discrimination accuracy in the GCKD dataset (PROGRES-CKD-6, CKD stages 4-5, AUC = 0.91 (95\%CI 0.86-0.97); PROGRES-CKD-24, CKD stage 3-5, AUC $=0.85$ (95\%CI 0.83-0.88)).

Evaluation of ratios of observed risk across quintiles of predicted risk indicated that the model best discriminated low and high-risk patients compared to those classified in the central quintile or risk score distribution (Figure 3). 
(A)

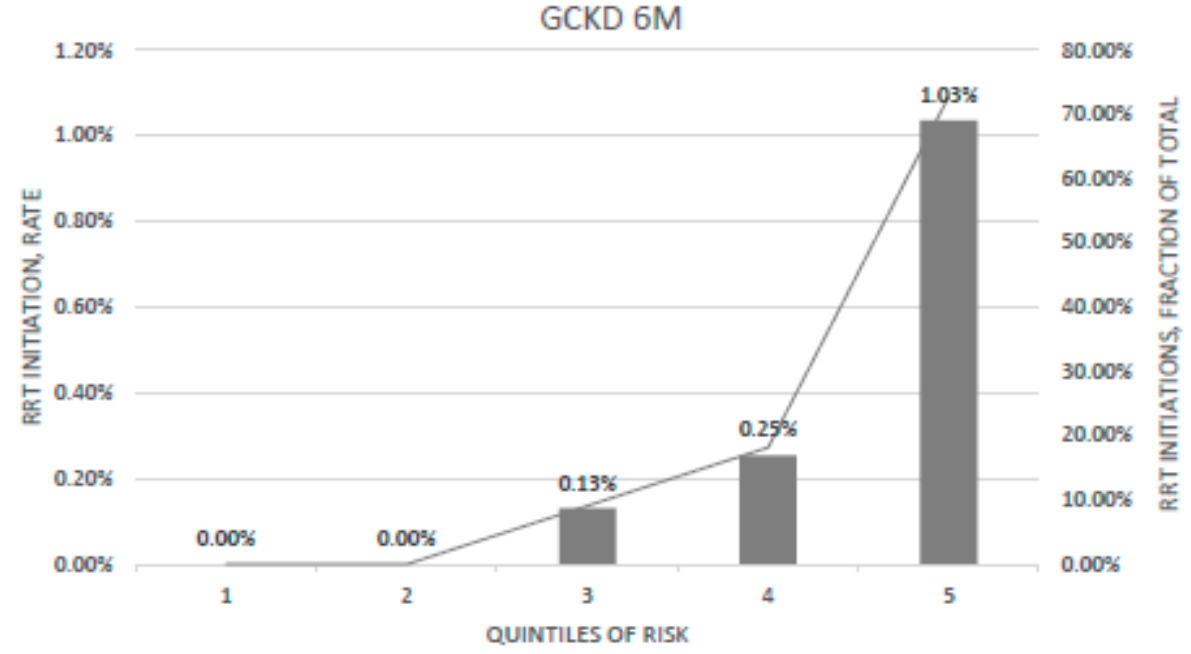

(B)

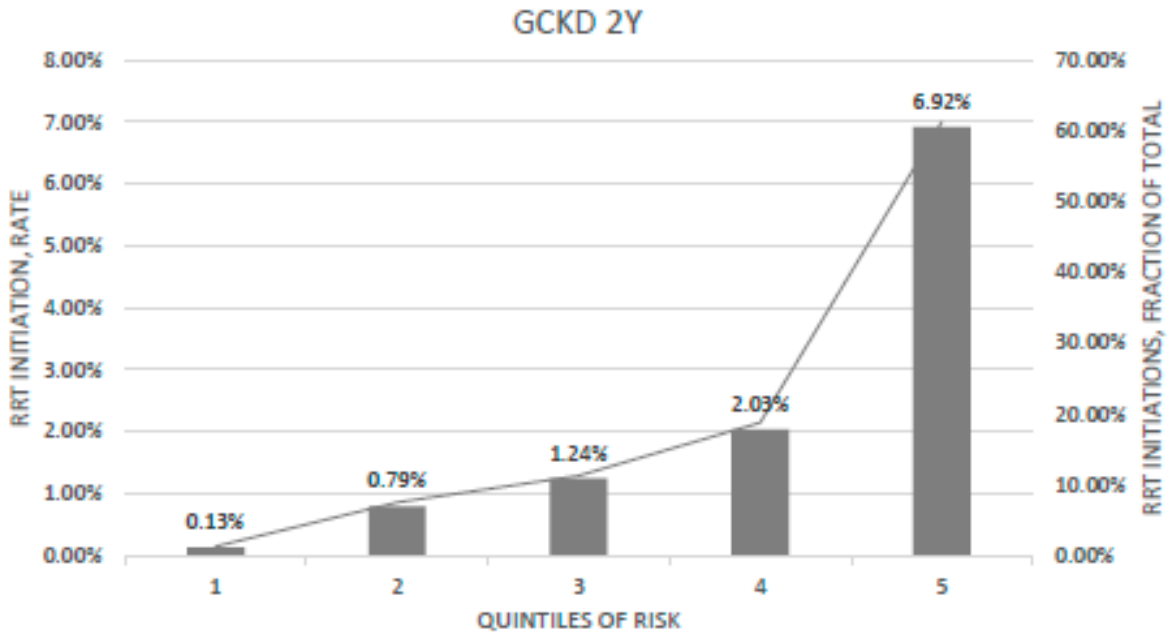

Figure 3. Calibration of (A) PROGRES-CKD-6, and (B) PROGRES-CKD-24 in the GCKD cohort. Bar graph denotes the incidence of RRT initiation events observed in each quintile of risk (left axis); line graph denotes the fraction of RRT initiation events in each quintile with respect to the total number of RRT initiation events (right axis). Endpoint horizons: 6 months for PROGRES-CKD-6; 24 months for PROGRES-CKD-24.

\subsection{Comparison with KFRE Performance}

Table 3 shows the comparison in discrimination accuracy between PROGRES-CKD and KFREs equations. Since KFREs equations are computable only for complete information cases, patients with missing data were listwise deleted from this analysis. Given the large amount of missing information for ACR, we converted timed proteinuria assays (proteinuria g/24 h) into ACR when available. The conversion was based on a published correspondence table (Supplementary Material). 
Table 3. Comparison between discrimination ability of (A) PROGRES-CKD-6 and (B) PROGRES-CKD-24 and that of Tangri's Kidney Failure Risk Equations (KFREs) in the FMC and the GCKD cohort. The two scores were computed considering only complete cases (column "Effective sample size"), while patients with missing data were not included in the analysis. Endpoint horizons: 6 months for PROGRES-CKD-6; 24 months for PROGRES-CKD-24. Imputation method: Listwise. Non-inferiority was defined as AUC $<0.05$, while superiority was set at $\triangle \mathrm{AUC} \geq 0.05$. * Delta AUC: AUC of Tangri's KFRE-AUC of PROGRES-CKD model.

\begin{tabular}{|c|c|c|c|c|c|c|}
\hline Model & Validation Cohort & Comparator Model & AUC PROGRES-CKD & Delta AUC* & $p$-Value & Effective Sample Size \\
\hline \multicolumn{7}{|c|}{ PROGRES-CKD-6 } \\
\hline \multicolumn{7}{|c|}{ FMC NephroCare } \\
\hline & & 4VAR & 0.90 & -0.012 & 0.3255 & 927 \\
\hline & & 6VAR & 0.90 & -0.016 & 0.2220 & 927 \\
\hline \multicolumn{7}{|c|}{ GCKD } \\
\hline & & 4VAR & 0.91 & -0.146 & 0.0016 & 459 \\
\hline & & 6VAR & 0.91 & -0.149 & 0.0013 & 459 \\
\hline \multicolumn{7}{|c|}{ PROGRES-CKD-24 } \\
\hline & \multicolumn{6}{|l|}{ FMC NephroCare } \\
\hline & & 4VAR & 0.87 & 0.020 & 0.0483 & 1081 \\
\hline & & 6VAR & 0.87 & 0.018 & 0.0888 & 1081 \\
\hline \multicolumn{7}{|c|}{ GCKD } \\
\hline & & 4VAR & 0.85 & 0.030 & 0.0105 & 3999 \\
\hline & & 6VAR & 0.85 & 0.027 & 0.0246 & 3999 \\
\hline
\end{tabular}

Based on the superiority test criteria, the discrimination accuracy of PROGRES-CKD-6 was greater than KFRE equations for short term RRT risk among stage 4-5 CKD patients (Table 3). PROGRES-CKD-24 discrimination was not inferior to that of the gold standard algorithms (Table 3).

\subsection{Potential Impact Simulation}

A potential impact study compared the risk of KRT estimated by nephrologists with those calculated by PROGRES-CKD-24 and investigated the potential incremental efficiency of using PROGRES-CKD compared to physicians' assessments to inform referral to an intensified multidisciplinary prevention program to delay progression to ESKD.

Table 4 reports ratings of CKD progression risks provided by either physicians or the prediction model. In the evaluation sample, 25 patients required KRT within 2 years, while 53 patients did not reach the study endpoint. PROGRES-CKD-24 had excellent discrimination within this dataset $(\mathrm{AUC}=0.96)$, while experts' ratings demonstrated good discrimination (average AUC $=0.79$ ), with average sensitivity $=0.64$ and average specificity $=0.85$ at the optimal cut-off point (score $>6$ ). Therefore, experts were less discriminative of endpoint occurrence compared to PROGRES-CKD-24 ( $\triangle \mathrm{M}-\mathrm{E}=0.17, p=0.005)$. The correlation of physicians' ratings with PROGRES-CKD-24 ratings was moderate $(\mathrm{r}=0.50$, $p<0.01$ ); furthermore, experts showed different abilities to discriminate patients' risk. (Table 4).

Table 4. PROGRES-CKD-24 and Experts' ratings of CKD progression risk.

\begin{tabular}{lccccc}
\hline & & \multicolumn{4}{c}{ Experts } \\
\hline & PROGRES-CKD-24 & Expert 1 & Expert 2 & Expert 3 & Expert 4 \\
\hline AUC & 0.96 & 0.84 & 0.72 & 0.86 & 0.76 \\
Sensitivity & 0.76 & 0.80 & 0.50 & 0.75 & 0.60 \\
Specificity & 0.96 & 0.84 & 0.89 & 1.00 & 0.82 \\
\hline
\end{tabular}

Figure 4 shows the results of our impact simulation. Based on the experts' ratings $(\mathrm{PPV}=17 \%$; FOR $=2 \%), n=1725(17.3 \%)$ patients would be assigned to the high-risk category, while $n=8275(82.8 \%)$ would be recommended to the standard care program (Figure 4, panel A). Based on the assumptions set for the simulation exercise (i.e., ESKD overall incidence without intervention: 2.3 events/100 patient-years; ESKD risk is reduced by $50 \%$ in the intensified intervention group) there would be 362 ESKD events overall. 
Therefore, in this scenario, physicians' referral to the intensified program would delay 98 ESKD cases (i.e., an Overall Program Effect Size of 1.27). The number of patients needed to treat would be NNT = 18 (Figure 4, panel D). Conversely, risk stratification by PROGRESCKD-24 (PPV $=48 \%$; FOR $=1.2 \%)$ leads to referral of $n=732(0.73 \%)$ patients to intensified intervention (Figure 4, panel B). In this case, 117 ESRD events would be prevented, i.e., an Overall Program Effect Size of 1.36. The number needed to treat would be NNT $=6$ (Figure 4, panel D). Finally, under a hypothetical risk averse policy that would refer all stage 3 CKD patients to the intensified program, 153 ESRD events would be prevented with NNT = 65 (Figure 4, panel C).

A

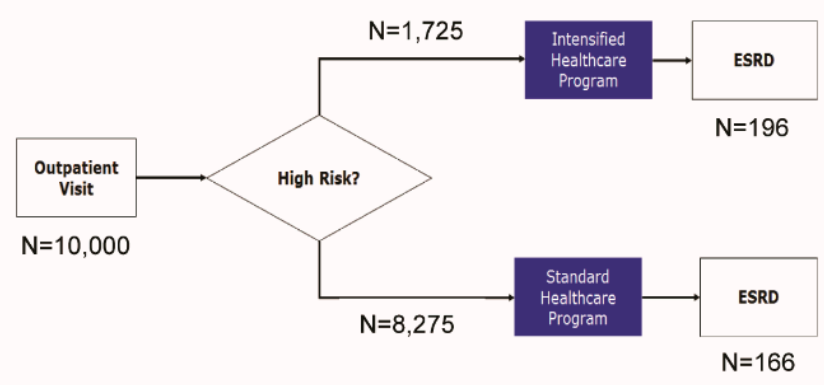

C

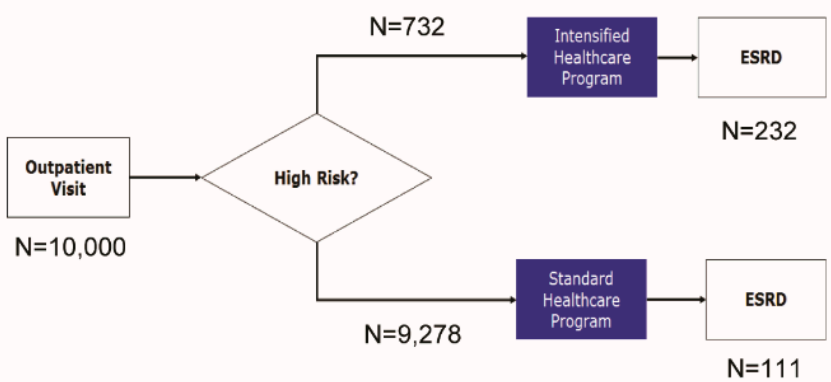

B

ESRD cases after 24 months

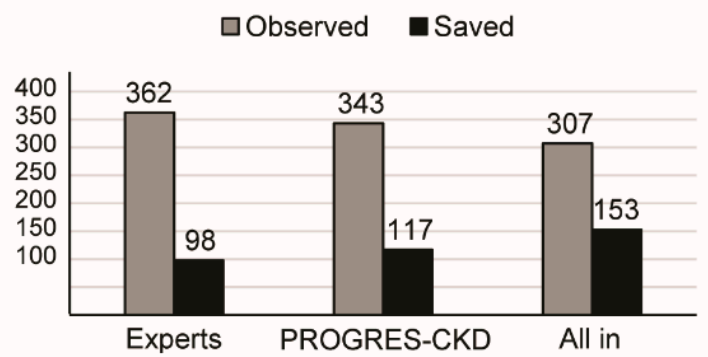

D

\section{Number needed to treat}

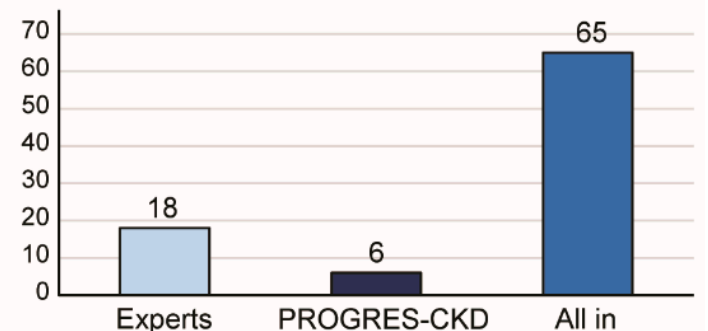

Figure 4. Potential impact simulation of PROGRES-CKD-24 implementation in a hypothetical CKD cohort. Flowcharts showing patients' referral to intensified intervention programs based on (A) experts' ratings, and (B) PROGRES-CKD scores; (C) Number of ESKD events within 24 months: both observed and saved cases are shown; D) Number of patients needed to treat to save 1 patient; "all-in strategy" involves referral of all stage 3 patients to the intensified healthcare program. Abbreviations: ESKD, end-stage kidney disease; NNT, Number needed to treat.

\section{Discussion}

The present study reports the derivation and validation of the PROGRES-CKD algorithm in two independent cohorts of non-dialysis dependent CKD patients. Discrimination accuracy of PROGRES-CKD was excellent for both the short-term prediction horizon (6 months) and the long-term prediction horizon (24 months).

Of note is the fact that PROGRES-CKD-6 and PROGRES-CKD-24 had reproducible discrimination accuracy in both validation studies. The FMC NephroCare cohort included real-world clinical data of stage 3-5 CKD patients from 15 countries (Europe, SouthAmerica, Africa), while the GCKD study is a prospective CKD cohort study recruiting a wider range of NDD-CKD patients with moderate GFR impairment in Germany [21]. Given the substantial differences between the two cohorts in geographical area of recruitment (international vs. national), inclusion/exclusion criteria, and data collection strategies (real-world vs. pre-specified protocol), the observed consistency in discrimination and calibration corroborates the generalizability of PROGRES-CKD across different CKD subpopulations and clinical settings. 
To further characterize PROGRES-CKD accuracy, we compared its discrimination performance against KFREs which were extensively validated in different CKD patient populations $[11,17,18]$ and are routinely used in clinical practice. PROGRES-CKD was as accurate as KFREs for 24-month prediction in both validation cohorts and more accurate for 6-month forecasting in the GCKD study. Even though the two algorithms showed comparable performance in long-term prediction, the KFRE risk score could not be computed in a vast share of patients of the FMC NephroCare cohort because of missing information of key input variables (Figure 5). Conversely, PROGRES-CKD was available for all patients due to accurate handling of missing variables inherent to naïve Bayes classifiers (Figure 5) [36]. In fact, PROGRES-CKD potentially incorporates input from as many as 32 clinical parameters, yet its prediction can be computed with any subset of information. Therefore, PROGRES-CKD performance remained stable even for patients with many missing parameters representative of a real-world clinical practice setting. Furthermore, by assessment of VOI metrics, PROGRES-CKD allows the graphical representation of the uncertainty around prediction due to missing data. Given that VOI metrics are calculated for each missing clinical parameter within the patient's health records, they can be used to rank the potential prognostic benefit of additional diagnostic testing or biomarker assays for patients with incomplete medical data. These peculiar features of PROGRES-CKD significantly increase its clinical usability in that they enable to address the problem of missing predictors in real-world data [17] by exploiting the full wealth of information collected in routine clinical practice.

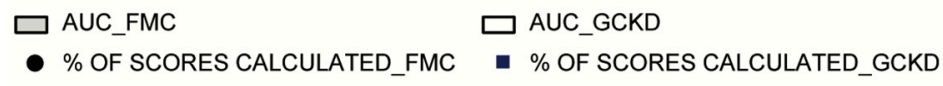

\section{A $\quad$ RRT prediction within 6 months}

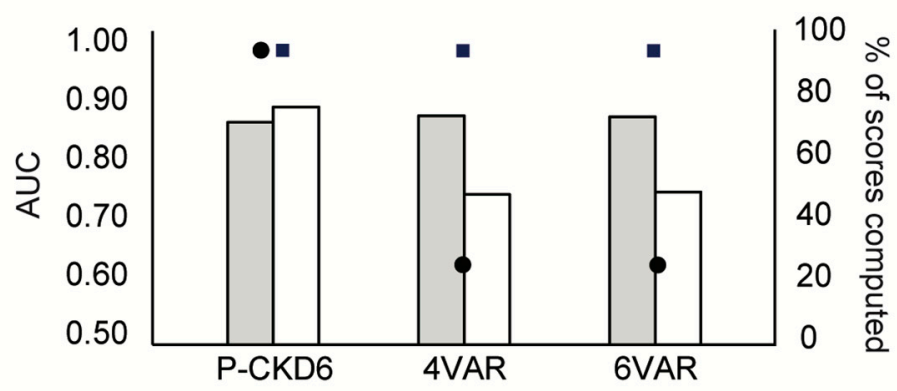

B $\quad$ RRT prediction within 24 months

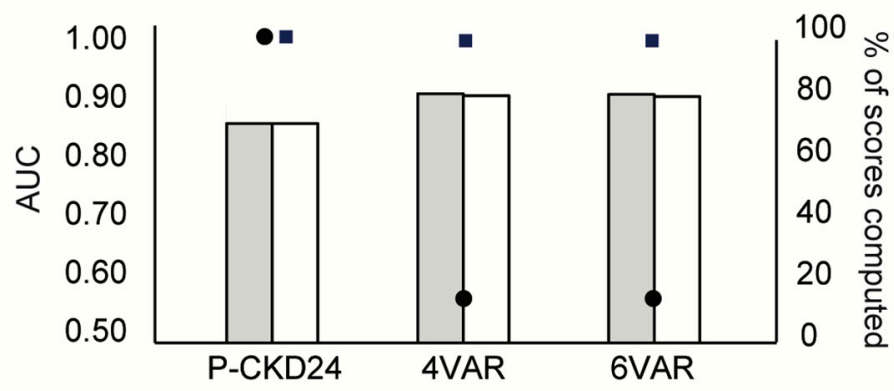

Figure 5. Discrimination ability of PROGRES-CKD and KFREs and percentage of computed scores by each prediction tool. Only cases with complete medical information were included in this analysis. (A) RRT prediction within 6 months; (B) RRT prediction within 24 months. Bars denote AUC (left yaxis), while dots denote the percentage of computed scores on the total number of recruited patients in each cohort (right y-axis). Abbreviations: P-CKD6, PROGRES-CKD-6; P-CKD24, PROGRES-CKD-24; 4VAR, KFRE 4 variables; 6VAR, KFRE 6 variables. 
One additional advantage of NBCs such as PROGRES-CKD over traditional equationbased prediction tools rest in their ability to generate personalized, patient-specific impact metrics representing the relative contribution of each predictor to a patient's risk. Impact metrics can be used to estimate the potential impact of interventions addressing modifiable risk factors. This has important implications for patient care, since there can be considerable heterogeneity in underlying diseases, demographics, co-morbidities, and risk for progression among CKD patients and, consequently, optimal intervention strategies might deviate between patients with the same overall risk estimate depending on their individual high impact risk parameters. Therefore, both VOI and impact metrics could help physicians within their decision-making processes in tailoring interventions according to each individual patient's needs and characteristics [37]. Adoption of a more personalized clinical approach would lead not only to improved CKD clinical management (targeted diagnostic and treatment investigations with minimum adverse events and maximum efficacy, and consequently increased adherence to treatment), but it could also contribute towards optimizing the utilization of healthcare resources. In fact, ranking clinical parameters by their impact on risk score computation helps physicians' reasoning on priority and enables strategic and rational formulation of therapeutic plans considering both patient/disease-related factors and resource availability.

One specification of PROGRES-CKD allows the identification of patients whose kidney function is more likely to deteriorate within 6 months, a feature enabling timely referral to vascular access creation services and transition management $[38,39]$. The potential advantages of accurate short-term progression are two-fold. Patients starting on chronic dialysis with an arteriovenous fistula (AVF) rather than catheter have improved clinical outcomes in terms of survival, hospitalization, and complications [40]. On the other hand, inappropriate AVF creation in stage 4 and 5 patients who do not rapidly progress to KF is associated with complications and premature loss of patency [38].

Accurate risk prediction is a challenging task for physicians in real-world clinical practice, due to a number of disease, clinician, and organization related factors, including: inherent heterogeneity and variability in CKD progression rates [41,42], incomplete information, unrecognized case ambiguity, overconfidence leading to reduced analytical scrutiny, wrong perception of average population risk, over-generalization, fatigue, working overload, aging, altered affect impairing executive memory, switch of analytic scrutiny, and inexperience [43-48]. Therefore, readily available risk scores which prove to be accurate, generalizable to a wide array of CKD subpopulations and settings, and robust to missing data patterns observed in real-life applications may considerably assist clinical decision making, particularly when providing the opportunity to simulate the impact of interventions to individual patient cases.

In order to estimate the potential impact of improved prognostication around CKD progression on process outcomes, clinical outcomes, and costs [38,49], we conducted a simplified simulation using PROGRES-CKD as a patient stratification system for referral to intensified prevention programs for non-dialysis dependent (NDD)-CKD patients. In our simulation, risk estimates provided by either PROGRES-CKD or nephrology experts were used to stratify CKD patients. Subjects assigned to the "high-risk" category are referred to an intensified healthcare program aimed at reducing the risk of CKD progression. Our analysis suggested that PROGRES-CKD-driven referral to the intensified program would be more effective and largely more efficient than referral patterns determined by both healthcare expert risk assessment and an "all-in strategy" (i.e., all patients are referred to the intensified healthcare program when they reach stage $3 \mathrm{CKD}$ ). Therefore, personalized, risk-based referral may improve the efficiency of healthcare systems by enhancing the appropriateness of resource allocation in terms of direct expenditures and staff utilization. Personalized referral, however, is not just a matter of mere efficiency. In fact, inappropriate referral to the intensified intervention would involve unnecessary medicalization with greater risks of adverse events, impoverishment of quality of life even in people with a very low risk of progression, increased rate of therapeutic fatigue, and reduced adherence. 
Conversely, accurate and reliable patient stratification helps physicians and healthcare providers balance individual patient needs with overall resource utilization, ultimately leading to more effective care for both the individual patient and the population [50].

\section{Limitations}

Validation of risk score should be considered a continuous process of generalization tests rather than a single experiment. While the performance of PROGRES-CKD was stable in both well-conducted longitudinal cohort studies (i.e., GCKD) and historical cohorts of real-life practice (i.e., FMC NephroCare), evidence concerning PROGRES-CKD robustness with real-world-representing clinical practices outside FMC NephroCare is still missing. For this reason, PROGRES-CKD undergoes a periodical process of performance monitoring while external cohorts for validation exercises are actively sought for.

\section{Conclusions}

The Prognostic Reasoning System for CKD patients (PROGRES-CKD) demonstrated excellent discrimination accuracy in two independent cohorts of NDD-CKD patients. The underlying models provide accurate prediction for both 24 and 6 months KRT risk. Contrary to traditional equation-based algorithms which cannot be applied to a large proportion of patients with incomplete data, PROGRES-CKD extends to all patients and allows explicit assessment of prediction robustness in case of missing values for key risk factors. Furthermore, PROGRES-CKD enhances prognostic reasoning by providing patientspecific impact metrics representing the relative contribution of each predictor to a patient's risk and can be used to estimate the potential impact of tailored interventions in addressing individual and modifiable risk factors. While PROGRES-CKD-24 may contribute to efficient and effective referral to intensified prevention programs for NDD-CKD patients, prediction of short-term outcomes (PROGRES-CKD-6) can be a key enabler of timely AVF creation and transition management. Given these results, both PROGRES-CKD algorithms reported here have the potential to advance current standards in routine CKD risk estimation, patient stratification, and individualizing interventions.

Supplementary Materials: The following supplements are available online at https://www.mdpi. com/article/10.3390/ijerph182312649/s1, Supplementary Table S1. List of ICD10 codes used to abstract comorbidity variables; Supplementary Table S2. Urin protein Conversion table; Supplementary results. Case study; Supplementary Figure S1. Graphical output of PROGRES-CKD.

Author Contributions: F.B. contributed to study concept, design, statistical analysis, interpretation of results, manuscript drafting, and approved the final version of the manuscript; C.L., performed literature search, contributed to interpretation of results, drafted the first version of the manuscript, and approved the final version of the manuscript; J.I.T. contributed to interpretation of results and drafted the first version of the manuscript and approved the final version of the manuscript; J.N. contributed to data acquisition, interpretation of results, and reviewed and approved the final version of the manuscript; H.M. contributed to data acquisition, interpretation of results, and reviewed and approved the final version of the manuscript; M.S. (Matthias Schmid) contributed to data acquisition, interpretation of results, and reviewed and approved the final version of the manuscript; B.B. contributed to data acquisition, interpretation of results, and reviewed and approved the final version of the manuscript; U.T. performed literature search, project conceptualization and project administration, and reviewed and approved the final version of the manuscript; M.S. (Markus Schneider), contributed to data acquisition, interpretation of results, and reviewed and approved the final version of the manuscript; U.T.S. contributed to data acquisition, interpretation of results, and reviewed and approved the final version of the manuscript; C.B. contributed to study concept, interpretation of results, and approved the final version of the manuscript; C.M. contributed to interpretation of results, and approved the final version of the manuscript; S.S. (Sonja Steppan) contributed to interpretation of results, and approved the final version of the manuscript; K.-U.E. contributed to data acquisition, interpretation of results, and reviewed and approved the final version of the manuscript; S.S. (Stefano Stuard) contributed to interpretation of results, and reviewed and approved the final version of the manuscript; L.N., contributed to study concept, design, statistical analysis, interpretation of results, manuscript drafting, and approved the final version 
of the manuscript. All authors have read and agreed to the published version of the manuscript. Authors confirm that they had full access to all the data in the study and accept responsibility of submission for publication.

Funding: This research was funded by Fresenius Medical Care Deutschland GmbH.

Institutional Review Board Statement: The study was approved by the ethics committees of all participating institutions and registered in the national registry for clinical studies (DRKS 00003971).

Informed Consent Statement: No patients' personal information has been used for the present study since all input data for modeling were aggregated statistics.

Data Availability Statement: We are unable to share the raw clinical data of the FMC NephroCare because data sharing would violate the terms and conditions under which Fresenius Medical Care acquired the data. Data from the GCKD study are not publicly available. External collaborators with a specific research proposal can access deidentified participant data only after review and approval of their proposal by the steering committee.

Acknowledgments: The GCKD study was supported by the German Ministry of Education and Research (Bundesministerium für Bildung und Forschung, FKZ 01ER 0804, 01ER 0818, 01ER 0819, 01ER 0820, and 01ER 0821), KfH Foundation for Preventive Medicine, Innovative Medicines Initiative 2 Joint Undertaking (BEAt-DKD, grant number 115974), and corporate sponsors (www.gckd.org).

Conflicts of Interest: The results presented in this paper have not been published previously in whole or part, except in abstract format. L.N., J.I.T., F.B., S.S. (Sonja Steppan), S.S. (Stefano Stuard), C.M., C.B., U.T. are full time employees at Fresenius Medical Care. C.L. provided medical writing services on behalf of Fresenius Medical Care. H.M. reports grants from KfH Foundation of Preventive Medicine, and grants from German ministry of Education and Research. M.S. (Matthias Schmid) reports grants from Fresenius Medical Care during the conduct of the study. B.B. reports grants from the Federal Ministry of Education and Research (Bundesministerium für Bildung und Forschung (www.bmbf.de), FKZ 01ER 0804, 01ER 0818, 01ER 0819, 01ER 0820 und 01ER 0821), and grants from Foundation for Preventive Medicine of the KfH (Kuratorium für Heimdialyse und Nierentransplantation e.V.Stiftung Präventivmedizin; www.kfh-stiftung-praeventivmedizin.de). MSchneider reports grants from Fresenius Medical Care outside the submitted work. K.-U.E. reports grants from: Astra Zeneca, Bayer, Fresenius Medical Care, Vifor, and Amgen during the conduct of the study; personal fees from Akebia, Astellas, Astra Zeneca, Bayer, and Boehringer Ingelheim; and grants from Genzyme, Shire, and Vifor outside the submitted work. J.N. has no conflicts of interest to disclose. U.T.S. has no conflicts of interest to disclose.

\section{References}

1. Peng, S.; He, J.; Huang, J.; Lun, L.; Zeng, J.; Zeng, S.; Zhang, L.; Liu, X.; Wu, Y. Self-management interventions for chronic kidney disease: A systematic review and meta-analysis. BMC Nephrol. 2019, 20, 142. [CrossRef] [PubMed]

2. Navaneethan, S.D.; Shao, J.; Buysse, J.; Bushinsky, D.A. Effects of treatment of metabolic acidosis in CKD: A systematic review and meta-analysis. Clin. J. Am. Soc. Nephrol. 2019, 14, 1011-1120. [CrossRef] [PubMed]

3. Heerspink, H.J.L.; Greene, T.; Tighiouart, H.; Gansevoort, R.T.; Coresh, J.; Simon, A.L.; Chan, T.M.; Hou, F.F.; Lewis, J.B.; Locatelli, F.; et al. Change in albuminuria as a surrogate endpoint for progression of kidney disease: A meta-analysis of treatment effects in randomised clinical trials. Lancet Diabetes Endocrinol. 2019, 7, 128-139. [CrossRef]

4. Inker, L.A.; Heerspink, H.J.L.; Tighiouart, H.; Levey, A.S.; Coresh, J.; Gansevoort, R.T.; Simon, A.L.; Ying, J.; Beck, G.J.; Wanner, C.; et al. GFR slope as a surrogate end point for kidney disease progression in clinical trials: A meta-analysis of treatment effects of randomized controlled trials. J. Am. Soc. Nephrol. 2019, 30, 1735-1745. [CrossRef] [PubMed]

5. Campbell, G.A.; Bolton, W.K. Referral and comanagement of the patient with CKD. Adv. Chronic Kidney Dis. 2011, 18, 420-427. [CrossRef] [PubMed]

6. Levin, A. Consequences of late referral on patient outcomes. Nephrol. Dial. Transplant. 2000, 15, 8-13. [CrossRef] [PubMed]

7. Bartmańska, M.; Więcek, A. Chronic kidney disease and the aging population. G. Ital. Nefrol. 2014, 36, 1-5. [CrossRef]

8. Fedewa, S.A.; McClellan, W.M.; Judd, S.; Gutiérrez, O.M.; Crews, D.C. The association between race and income on risk of mortality in patients with moderate chronic kidney disease. BMC Nephrol. 2014, 15, 136. [CrossRef]

9. Kadatz, M.J.; Lee, E.S.; Levin, A. Predicting Progression in CKD: Perspectives and Precautions. Am. J. Kidney Dis. 2016, 67, 779-786. [CrossRef]

10. Tangri, N.; Kitsios, G.D.; Inker, L.A.; Griffith, J.; Naimark, D.M.; Walker, S.; Rigatto, C.; Uhlig, K.; Kent, D.M.; Levey, A.S. Risk prediction models for patients with chronic kidney disease a systematic review. Ann. Intern. Med. 2013, 158, 596-603. [CrossRef] 
11. Ramspek, C.L.; de Jong, Y.; Dekker, F.W.; van Diepen, M. Towards the best kidney failure prediction tool: A systematic review and selection aid. Nephrol. Dial. Transplant. 2020, 35, 1527-1538. [CrossRef]

12. Dekker, F.W.; Ramspek, C.L.; Van Diepen, M. Con: Most clinical risk scores are useless. Nephrol. Dial. Transplant. 2017, 32, 752-755. [CrossRef]

13. Echouffo-Tcheugui, J.B.; Kengne, A.P. Risk Models to Predict Chronic Kidney Disease and Its Progression: A Systematic Review. PLoS Med. 2012, 9, e1001344. [CrossRef]

14. Collins, G.S.; Omar, O.; Shanyinde, M.; Yu, L.M. A systematic review finds prediction models for chronic kidney disease were poorly reported and often developed using inappropriate methods. J. Clin. Epidemiol. 2013, 66, 268-277. [CrossRef]

15. Tangri, N.; Stevens, L.A.; Griffith, J.; Tighiouart, H.; Djurdjev, O.; Naimark, D.; Levin, A.; Levey, A.S. A Predictive Model for Progression of Chronic Kidney Disease to Kidney Failure. JAMA 2011, 305, 1553-1559. [CrossRef]

16. Tangri, N.; Grams, M.E.; Levey, A.S.; Coresh, J.; Appel, L.J.; Astor, B.C.; Chodick, G.; Collins, A.J.; Djurdjev, O.; Raina Elley, C.; et al. Multinational Assessment of Accuracy of Equations for Predicting Risk of Kidney Failure ameta-analysis. JAMA 2016, 315, 164-174. [CrossRef]

17. Van Rijn, M.H.C.; van de Luijtgaarden, M.; van Zuilen, A.D.; Blankestijn, P.J.; Wetzels, J.F.M.; Debray, T.P.; Brand, J.A.J.G.V.D. Prognostic models for chronic kidney disease: A systematic review and external validation. Nephrol. Dial. Transplant. 2021, 36, 1837-1850. [CrossRef]

18. Kang, M.W.; Tangri, N.; Kim, Y.C.; An, J.N.; Lee, J.; Li, L.; Oh, Y.K.; Kim, D.K.; Joo, K.W.; Kim, Y.S.; et al. An independent validation of the kidney failure risk equation in an Asian population. Sci. Rep. 2020, 10, 1-10. [CrossRef]

19. Steil, H.; Amato, C.; Carioni, C.; Kirchgessner, J.; Marcelli, D.; Mitteregger, A.; Moscardo, V.; Orlandini, G.; Gatti, E. EuCliD ${ }^{\circledR}$ —A Medical Registry. Methods Inf. Med. 2004, 43, 83-88.

20. Merello Godino, J.I.; Rentero, R.; Orlandini, G.; Marcelli, D.; Ronco, C. Results from EuCliD ${ }^{\circledR}$ (European Clinical Dialysis Database): Impact of shifting treatment modality. Int. J. Artif. Organs 2002, 25, 1049-1060. [CrossRef]

21. Eckardt, K.U.; Bärthlein, B.; Seema, B.A.; Beck, A.; Busch, M.; Eitner, F.; Ekici, A.B.; Floege, J.; Gefeller, O.; Haller, H.; et al. The German Chronic Kidney Disease (GCKD) study: Design and methods. Nephrol. Dial. Transplant. 2011, 27, 1454-1460. [CrossRef] [PubMed]

22. Collins, G.S.; Reitsma, J.B.; Altman, D.G.; Moons, K.G.M. Transparent reporting of a multivariable prediction model for individual prognosis or diagnosis (TRIPOD): The TRIPOD statement. BMJ 2014, 350, g7594. [CrossRef] [PubMed]

23. Luo, W.; Phung, D.; Tran, T.; Gupta, S.; Rana, S.; Karmakar, C.; Shilton, A.; Yearwood, J.; Dimitrova, N.; Ho, T.B.; et al. Guidelines for developing and reporting machine learning predictive models in biomedical research: A multidisciplinary view. J. Med. Internet Res. 2016, 18, e323. [CrossRef] [PubMed]

24. McNally, R.J.; Heeren, A.; Robinaugh, D.J. A Bayesian network analysis of posttraumatic stress disorder symptoms in adults reporting childhood sexual abuse. Eur. J. Psychotraumatol. 2017, 8, 1341276. [CrossRef]

25. Khan, S.; Ghalib, M.R. A naive-bayes approach for disease diagnosis with analysis of disease type and symptoms. Int. J. Appl. Eng. Res. 2015, 10, 29005-29014.

26. Constantinou, A.C.; Yet, B.; Fenton, N.; Neil, M.; Marsh, W. Value of information analysis for interventional and counterfactual Bayesian networks in forensic medical sciences. Artif. Intell. Med. 2016, 66, 41-52. [CrossRef]

27. Kjærulff, U.B.; Madsen, A.L. Bayesian Networks and Influence Diagrams: A Guide to Construction and Analysis; Springer: New York, NY, USA, 2013.

28. Quan, H.; Sundararajan, V.; Halfon, P.; Fong, A.; Burnand, B.; Luthi, J.C.; Saunders, L.D.; Beck, C.A.; Feasby, T.E.; Ghali, W.A. Coding Algorithms for Defining Comorbidities in ICD-9-CM and ICD-10 Administrative Data. Med. Care 2005, 43, 1130-1139. [CrossRef]

29. Levey, A.S.; Stevens, L.A.; Schmid, C.H.; Zhang, Y.L.; Castro, A.F., III; Feldman, H.I.; Kusek, J.W.; Eggers, P.; Van Lente, F.; Greene, T.; et al. A New Equation to Estimate Glomerular Filtration Rate. Ann. Intern. Med. 2009, 150, 604-612. [CrossRef]

30. Oellgaard, J.; Gæde, P.; Rossing, P.; Persson, F.; Parving, H.-H.; Pedersen, O. Intensified multifactorial intervention in type 2 diabetics with microalbuminuria leads to long-term renal benefits. Kidney Int. 2017, 91, 982-988. [CrossRef]

31. Fogelfeld, L.; Hart, P.; Miernik, J.; Ko, J.; Calvin, D.; Tahsin, B.; Adhami, A.; Mehrotra, R.; Fogg, L. Combined diabetes-renal multifactorial intervention in patients with advanced diabetic nephropathy: Proof-of-concept. J. Diabetes Its Complicat. 2017, 31, 624-630. [CrossRef]

32. Peeters, M.J.; Van Zuilen, A.D.; Brand, J.A.J.G.V.D.; Bots, M.L.; Van Buren, M.; Dam, M.A.G.J.T.; Kaasjager, K.A.H.; Ligtenberg, G.; Sijpkens, Y.W.J.; Sluiter, H.E.; et al. Nurse Practitioner Care Improves Renal Outcome in Patients with CKD. J. Am. Soc. Nephrol. 2013, 25, 390-398. [CrossRef]

33. Linden, A. Measuring diagnostic and predictive accuracy in disease management: An introduction to receiver operating characteristic (ROC) analysis. J. Eval. Clin. Pract. 2006, 12, 132-139. [CrossRef]

34. Steyerberg, E.W.; Vickers, A.J.; Cook, N.R.; Gerds, T.; Gonen, M.; Obuchowski, N.; Pencina, M.J.; Kattan, M.W. Assessing the performance of prediction models: A framework for traditional and novel measures. Epidemiology 2010, 21, 128-138. [CrossRef]

35. Delong, E.R.; Delong, D.M.; Clarke-Pearson, D.L. Comparing the Areas under Two or More Correlated Receiver Operating Characteristic Curves: A Nonparametric Approach. Biometrics 1988, 44, 837-845. [CrossRef]

36. Nielsen, T.D.; Jensen, F.V. Bayesian Networks and Decision Graphs; Springer: New York, NY, USA, 2009. 
37. Sun, L.; Zou, L.-X.; Chen, M.-J. Make Precision Medicine Work for Chronic Kidney Disease. Med. Princ. Pract. 2016, 26 , $101-107$. [CrossRef]

38. Lerner, B.; Desrochers, S.; Tangri, N. Risk Prediction Models in CKD. Semin. Nephrol. 2017, 37, 144-150. [CrossRef]

39. Bargman, J.M. Timing of Initiation of RRT and Modality Selection. Clin. J. Am. Soc. Nephrol. 2015, 10, 1072-1077. [CrossRef]

40. Allon, M. Vascular access for hemodialysis patients: New data should guide decision making. Clin. J. Am. Soc. Nephrol. 2019, 14, 954-961. [CrossRef]

41. Webster, A.C.; Nagler, E.V.; Morton, R.L.; Masson, P. Chronic Kidney Disease. Lancet 2017, 389, 1238-1252. [CrossRef]

42. Levey, A.S.; Coresh, J. Chronic kidney disease. Lancet 2012, 379, 165-180. [CrossRef]

43. Jacoby, L.L.; McElree, B.; Trainham, T.N. Automatic influences as accessibility bias in memory and Stroop tasks: Toward a formal model. In Attention and Performance XVII: Cognitive Regulation of Performance: Interaction of Theory and Application; Elsevier: Amsterdam, The Netherlands, 1999; pp. 461-486.

44. Qin, S.; Hermans, E.J.; van Marle, H.J.F.; Luo, J.; Fernández, G. Acute Psychological Stress Reduces Working Memory-Related Activity in the Dorsolateral Prefrontal Cortex. Biol. Psychiatry 2009, 66, 25-32. [CrossRef]

45. Eva, K.W.; Norman, G.R. Heuristics and biases-A biased perspective on clinical reasoning. Med. Educ. 2005, 39, 870-872. [CrossRef]

46. Mumford, A.D.; Banning, A.P. Minimising delays to thrombolysis in patients developing acute myocardial infarction in hospital. Postgrad. Med. J. 1997, 73, 491-495. [CrossRef]

47. Croskerry, P.; Norman, G. Overconfidence in Clinical Decision Making. Am. J. Med. 2008, 121, 24-29. [CrossRef] [PubMed]

48. Croskerry, P. A Universal Model of Diagnostic Reasoning. Acad. Med. 2009, 84, 1022-1028. [CrossRef] [PubMed]

49. Moons, K.G.M.; Kengne, A.P.; Grobbee, D.E.; Royston, P.; Vergouwe, Y.; Altman, D.G.; Woodward, M. Risk prediction models: II. External validation, model updating, and impact assessment. Heart 2012, 98, 691-698. [CrossRef] [PubMed]

50. Moosa, M.R.; Maree, J.D.; Chirehwa, M.T.; Benatar, S.R. Use of the "accountability for reasonableness" approach to improve fairness in accessing dialysis in a middle-income country. PLOS ONE 2016, 11, e0164201. 